\title{
WIRE SEPARATION FROM AUTOMOTIVE SHREDDER RESIDUE
}

\author{
L. FABRIZI ${ }^{\mathrm{a}}$, T.P.R. DE JONG ${ }^{\mathrm{b}}$ and P. BEVILACQUA ${ }^{\mathrm{a}, *}$ \\ ${ }^{a}$ DICAMP, University of Trieste, Piazzale Europa n.1, 34127 Trieste, Italy; \\ ${ }^{\mathrm{b}}$ Department of Applied Earth Sciences, Delft University of Technology, \\ Mijnbouwstraat 120, $2628 \mathrm{RX}$ Delft, The Netherlands
}

(Received 14 July 2003; Accepted 23 July 2003)

\begin{abstract}
The investigation discussed in this article concerned the removal of wires and cables fraction contained in automotive shredder residue (ASR) through a newly invented device, called the 'nail roll'. Series of tests were carried out with three ASR samples of different origin and the influence of the setting parameters of the machine was analyzed. Other separation techniques and their combination with nail roll were also investigated, pursuing the objective of the increasing profit of the entire processing chain. The results indicate that the recovery index achievable by a very cost effective treatment is higher than $70 \%$ in the best case for all the three tested samples, while the grade index in the best case is $87 \%$ for the first sample, $70 \%$ for the second and $35 \%$ for the third. Nail roll efficiency is mostly dependent on the properties of the material treated and on its setting based on them.
\end{abstract}

Keywords: End of life vehicles; ASR; Wire separation; Nail roll

\section{INTRODUCTION}

Traditionally, scrap processing facilities were designed to recover metals, whereas other materials were collected in a residue fraction which is usually landfilled. The non-metallic residue streams generated at different stages of the car scrap processing are commonly known as automotive shredder residue (ASR). They can be divided into light and a heavy fractions: the former, called shredderfluff, is produced during or after shredding, the second arises as a mainly non-metallic reject material in different separation steps during the processing of the non-ferrous mix. The heavy fraction is mainly composed of massive to platy-shaped particles of plastics, rubber, stone, glass and copper wires. The composition of the light fraction is rather different: components are bulky fibers and foam, foils, plastics and rubber, some non-ferrous metals, copper wires, rust and dirt [1]. The average composition of ASR is shown in Table I [2].

*Corresponding author. E-mail: paolo.bevilacqua@dicamp.units.it 
TABLE I Average composition of ASR

\begin{tabular}{lr}
\hline & $\%$ \\
\hline Elastomers & 23 \\
Glass, ceramics & 13 \\
Chlorine free thermosets, form parts & 13 \\
Iron & 13 \\
Foam material & 7 \\
PVC & 6 \\
Other fibers and cover-materials & 6 \\
Other components & 5 \\
Wood, paper, cardboard & 4 \\
Aluminium & 3 \\
Other thermosets & 3 \\
Paint & 3 \\
Copper & 1 \\
\hline
\end{tabular}

Each year an estimated 40 million end of life vehicles (ELV) are treated by shredders all over the world. Since the average weight of a vehicle is 1 ton, about $75 \%$ of every vehicle is recovered and about $25 \%$ becomes ASR. Approximately 10 million tons of ASR is generated worldwide per year [3]. In Europe the number of ELV treated by shredders is approximately 10 million each year and up to 2.5 million tons of ASR are generated. ASR is mostly landfilled at present and it has, therefore, no economic value.

The directive 2000/53/EC of the European Parliament decreed that before 1 January 2006 for all ELV the reuse and recovery shall be increased to a minimum of $85 \%$ and the reuse and recycling to a minimum of $80 \%$ in weight per vehicle per year. Lower targets were decided for vehicles produced before 1 January 1980, but no less than $75 \%$ for reuse and recovery and $70 \%$ for reuse and recycling. Moreover, it was decided that before 1 January 2015 the reuse and recovery shall be increased to a minimum of $95 \%$ and the reuse and recycling of $85 \%$ per vehicle per year [4]. For these reasons technology has been developed in recent years to treat ASR by either thermal processing or mechanical separation. The technology aims at offering long-term solutions, but they usually suffer from both high investments and operational costs, requiring large feed volumes and a long-term stable supply for feasibility.

The presence of copper wires is sometimes a problem for mechanical processing of ASR, especially for the light fraction: fibers tend to interlock with them. Wire removal is attractive for this reason, because of the monetary value of copper and chlorine control. When substances containing chlorine are burned in uncontrolled conditions, toxic or carcinogenic dioxins and corrosion problems can arise [5].

\section{MARKET FOR REMOVED WIRES}

Copper is the third most used metal in the world, after steel and aluminium. World copper prices have steadily decreased since 1995 and in 1999 reached levels not seen since the early 1980s. As a result, copper scrap has been scarce in most world markets over the past four years [6]. Copper content in cars is rather high and tends to rise: more copper is being used as an alloying element for cast aluminium parts in the engine 
and transmission and the amount of sensors, wires, electric and electronic systems is increasing. As a result, copper cords can be found in shredder fluff.

In 2002 the total copper weight in vehicles was approximately $30 \mathrm{~kg}$, about $15 \mathrm{~kg}$ of which was formed by wire harnesses [7]. The average copper price was $1.3 € / \mathrm{kg}$. Therefore about $19.5 € /$ vehicle could have been recovered theoretically. Another metal often used in car wires is aluminium. Both of these metals can be recovered from wires contained in ASR.

Cable scrap attracts interest also for its plastics content: the two main plastics used in coated cables are PVC and polyethylene. Both are thermoplastics and can therefore be re-granulated for recycling. Eliminating wires from ASR is attractive as the problem of chlorine release can be avoided and a further disposal solution is made possible: generating energy (refuse derived fuel).

\section{STATE OF THE ART WIRE REMOVAL AND CABLE SCRAP PROCESSING}

\subsection{Wire Removal}

Wire removal is a process by which wires are concentrated into a product that has sufficient grade for further treatment by cable scrap processes at an optimized recovery. At the same time the wire content of the remaining material should be sufficiently low in order to avoid problems caused by wires in downstream processes. As an example inclined belts are used for concentration of copper-rich fractions (wires, circuit board fragments) from stones and other metals based on friction differences [8]. This device increases the concentration of wires in one of the fractions, but its selectivity is not high enough to obtain pure fractions.

TNO investigated a Hamos CUX L brush belt separator for shape separation of plastics/metal mixtures from pre-treated electronic scrap [9]. Grade of the copper was found to be approximately $70 \%$ at a recovery of between 75 and $80 \%$. The investigated capacity of $25 \mathrm{~kg} / \mathrm{h}$ may be considered rather low for industrial application in the processing of ASR.

\subsection{Cable Scraps Processing}

Once the wires are concentrated they need further treatment in order to separate the metals from the insulation. In the past this has been achieved by means of burning the insulation. However, cable burning is considered obsolete due to severe toxic emissions and environmental problems; it may still be practised in some countries. For the small diameter wires present in automotive scrap, cable stripping is not a satisfactory solution either [8]. Shredding by means of specifically designed cable granulating systems is an effective alternative [10]. After liberation the metals, mainly copper and aluminium, are separated from the insulation by means of density separation. In the past the Dryflo system was applied, which relies on separation in a dry fluidized bed of finely ground iron powder [11]. More recent applications typically apply air tables [8]. The remaining metals are separated into aluminium and copper after removal of the insulation and any iron residue that may still be present. 


\section{WIRE SEPARATION TECHNOLOGIES}

\subsection{Nail Roll}

The 'nail roll separator' was originally designed to recover the electric wires from mixed non-ferrous feed material, for example from a car shredder. It is a cylindrical roll to which the nails are attached in a regular chequered pattern. It rotates around its axis and vibrates in the axial direction. The ASR is fed on one side of the roll, which rotates in the counter direction. Wires are captured by the nails and discharged on the other side of the roll. The other particles pass through the nail forest and remain on the side from which the residue is fed $[12,13]$.

During tests with samples containing particles larger than the nail distance, two setting options were investigated: the distance between the nails and the introduction of an additional splitter next to the wire basket. In this way larger particles (mainly stones and plastics) do not mix with the wires, which are captured by the nails, but fall behind the second splitter (Figs. 1 and $2 \mathrm{a}-\mathrm{c}$ ).

The main operational variables that are critical for the separation are:

- horizontal distance, $d$, measured between the center of the roll and the end of the feeder,

- rotation speed, $w$, of the cylinder,

- feeder speed, $f$, the speed with which material is fed to the roll. It depends on the vibrational speed of the feeder,

- distance between the nails, $l$.

Feed

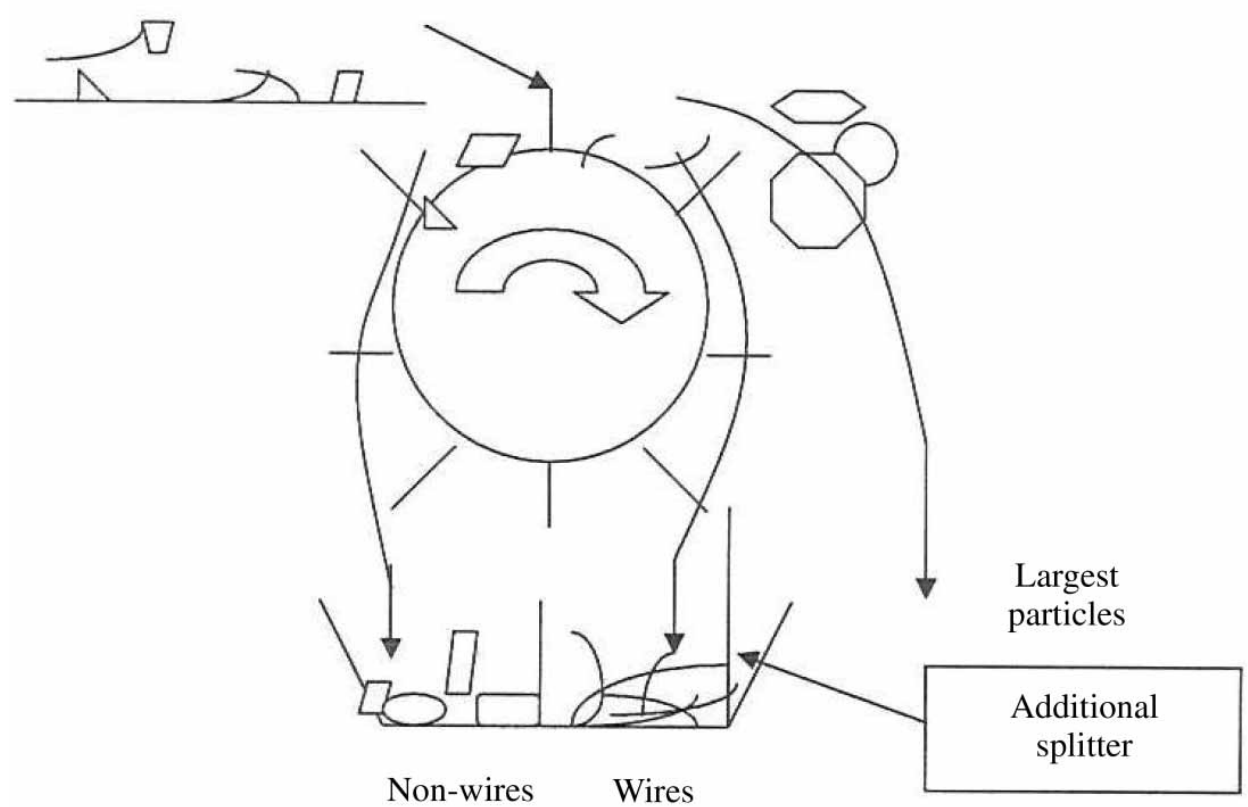

FIGURE 1 Principle of functioning of nail roll separator. 
The listed variables are shown in Fig. 2(a). They were considered during the study in order to find the best setting.

From a purely theoretical point of view, it can be observed that:

- A large horizontal distance should give higher grade and lower recovery. With a larger distance between the roll and the feeder most of the material falls before the roll and only some wires remain captured by the nails.

(a)

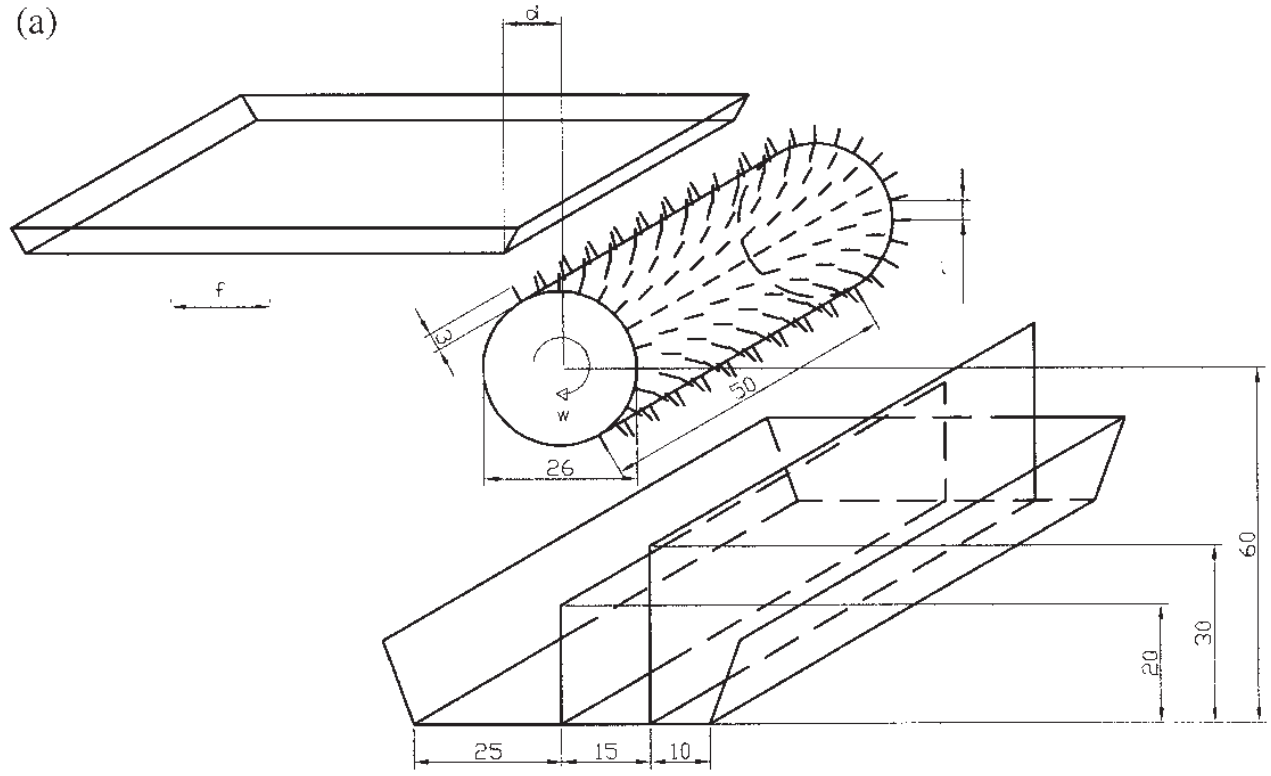

(b)

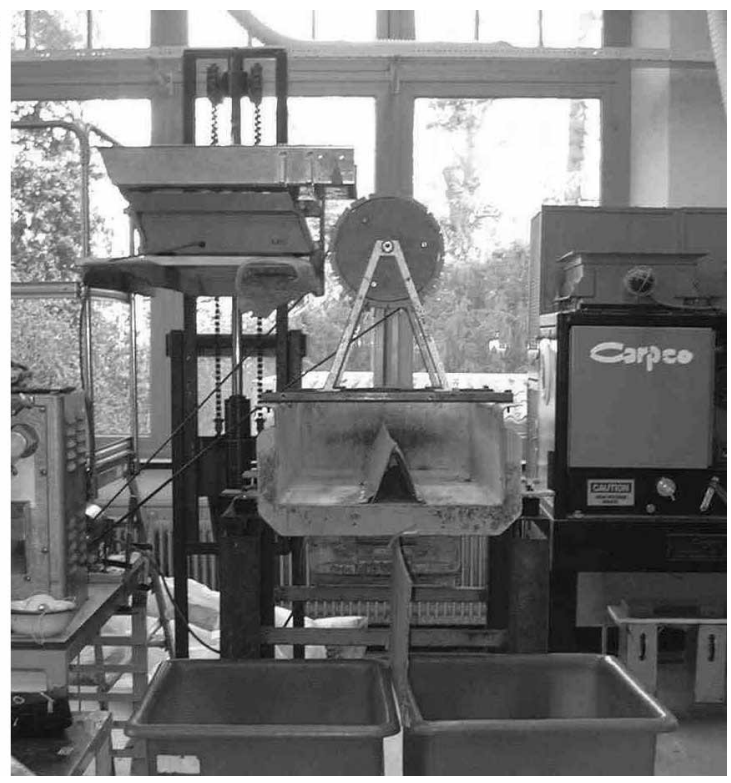

FIGURE 2 (a) The nail roll separator (dimensions are in centimetres); (b), (c) pictures. 
(c)

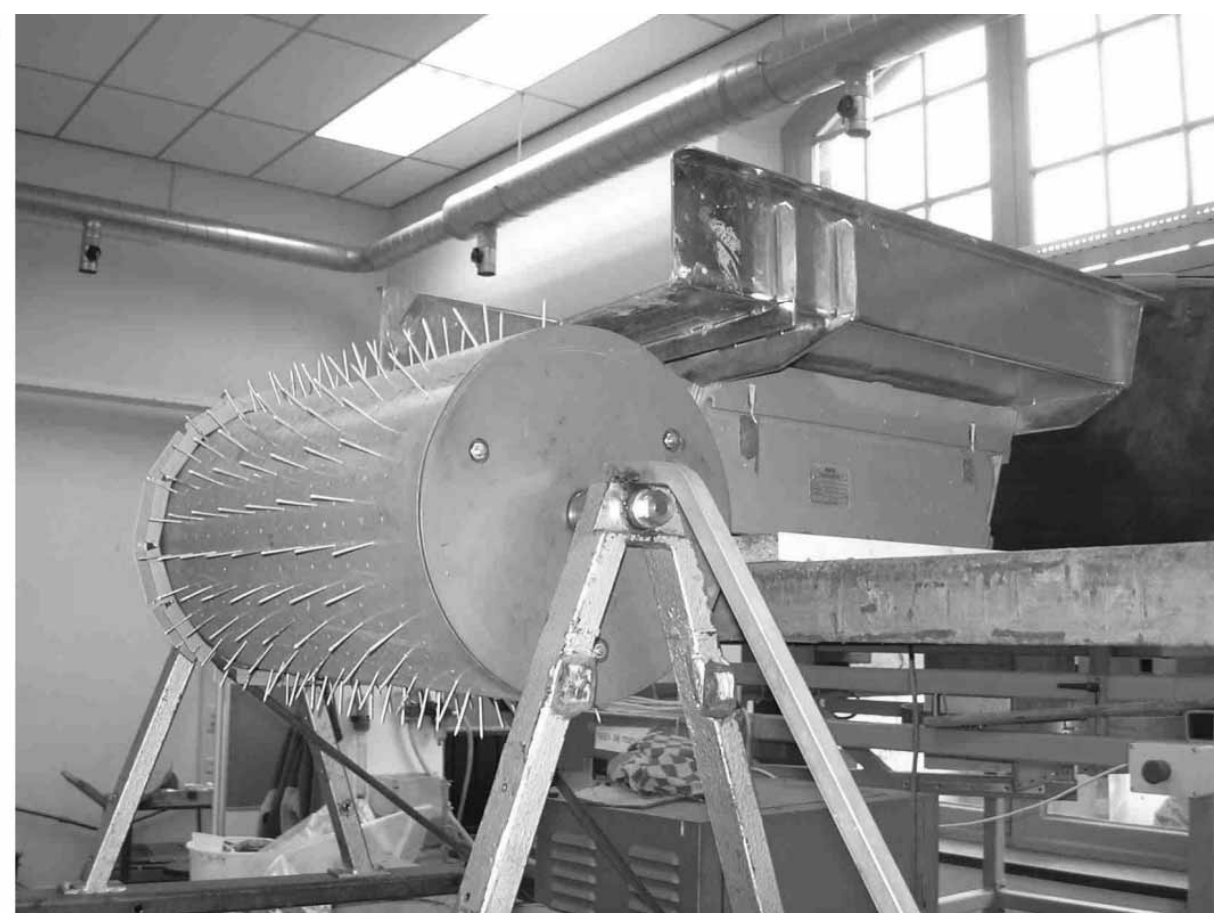

FIGURE 2 Continued.

- A high rotation speed is favors better recovery, but at the expense of the grade. This is because with a higher rotation speed the non-wires do not have enough time to pass the nails and so they end up in the wire fraction.

- With a high feeder speed the recovery should be good, but the grade should become lower as the speed increases: this is due to the fact that with high feeder speeds most of the material (wires and non-wires) ends up in the wire fraction. A high feeder speed is more economical for industrial applications.

- The optimum nail distance is related to the average size of the material to be processed. With a large nail distance we should obtain high recovery and grade for larger material.

\subsection{Other Investigated Techniques}

When the results obtained using only the nail roll proved unsatisfactory, other separation methods were applied as well. Such widening of the examination provides a more complete understanding of the complexities of the problem of wire separation from ASR.

\subsubsection{Heavy Medium Separation}

An average wire density of around $2000 \mathrm{~kg} / \mathrm{m}^{3}$ was measured by pycnometer. The rubber and the plastic insulation have a lower density than the metallic core. In principle the relatively high wire density could be sufficient to separate them from 
(a)

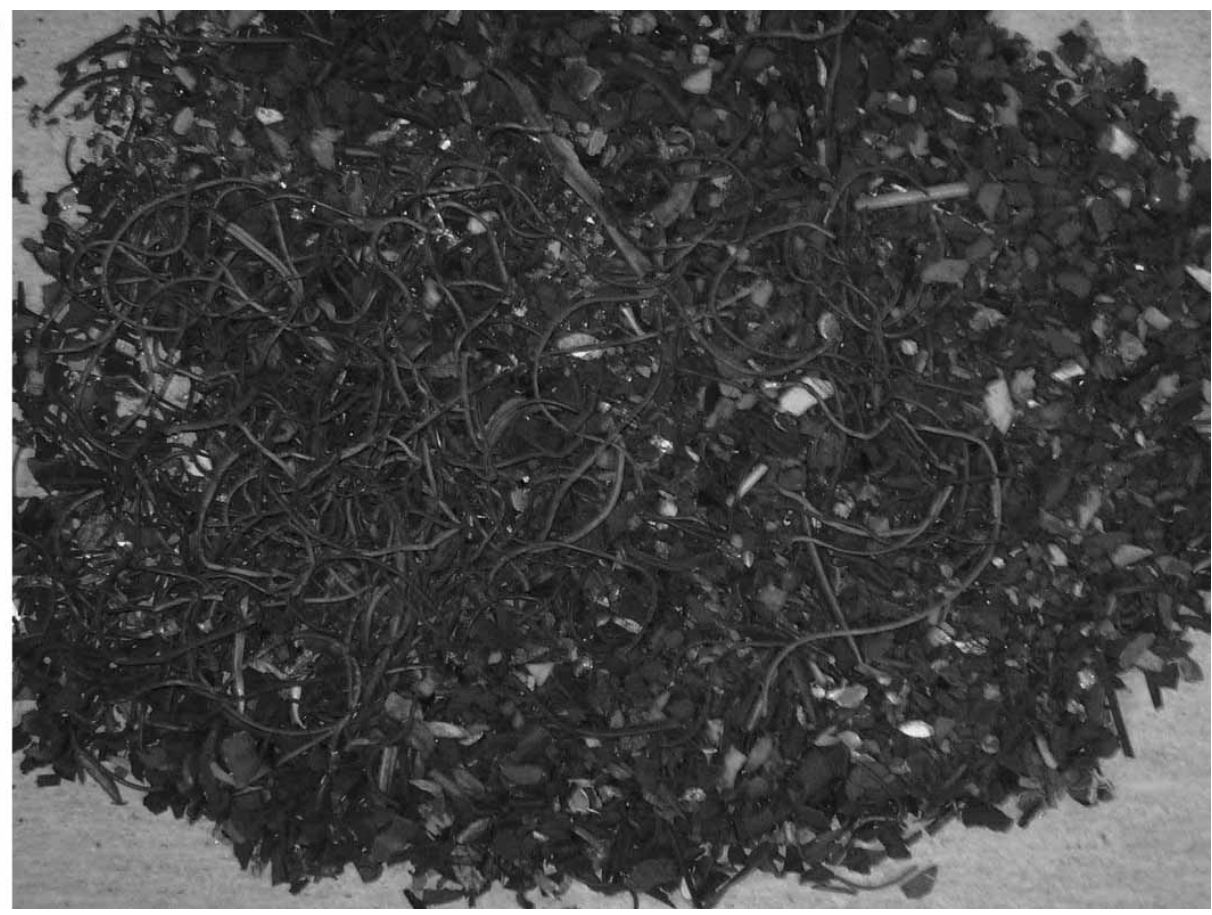

(b)

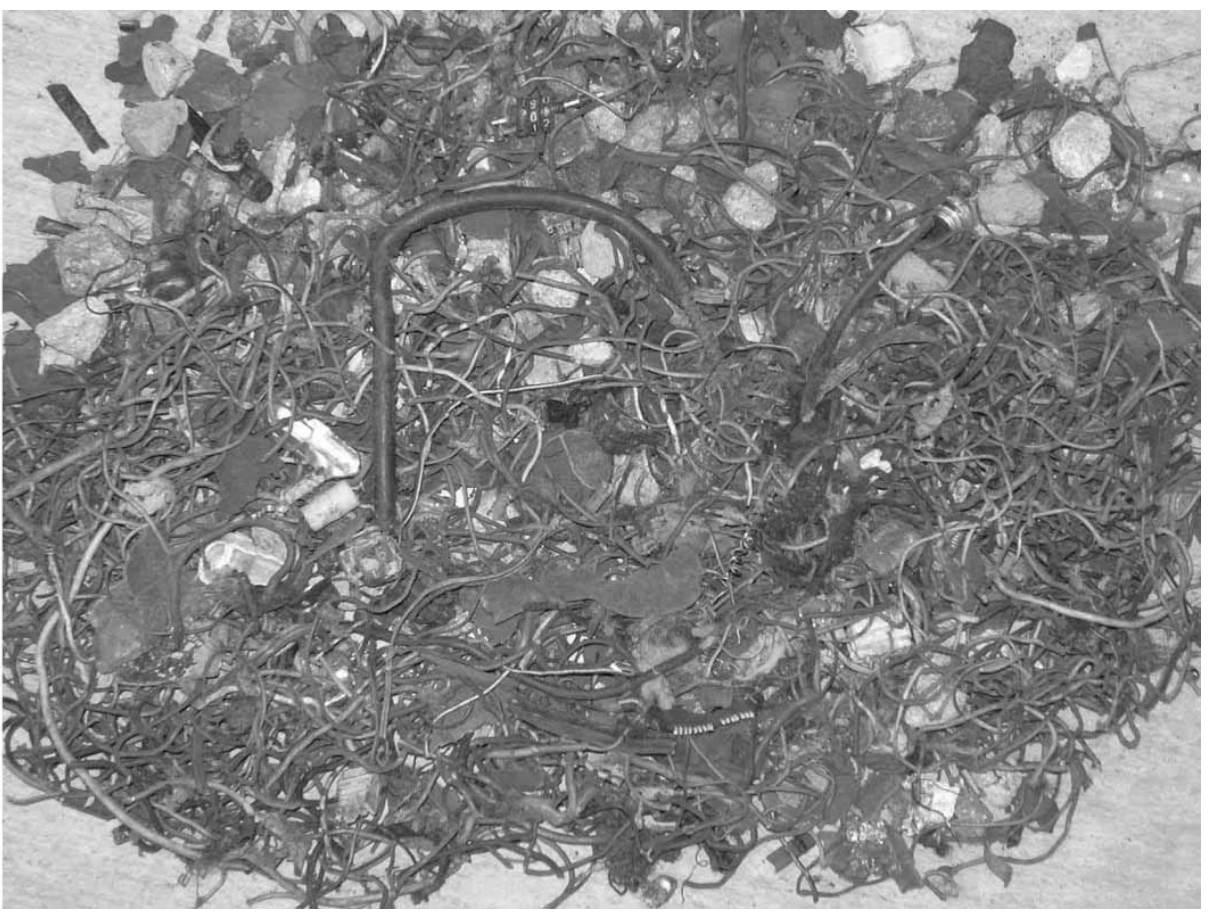

FIGURE 3 Images of the samples: (a) first sample; (b) second sample; (c) third sample. 
(c)

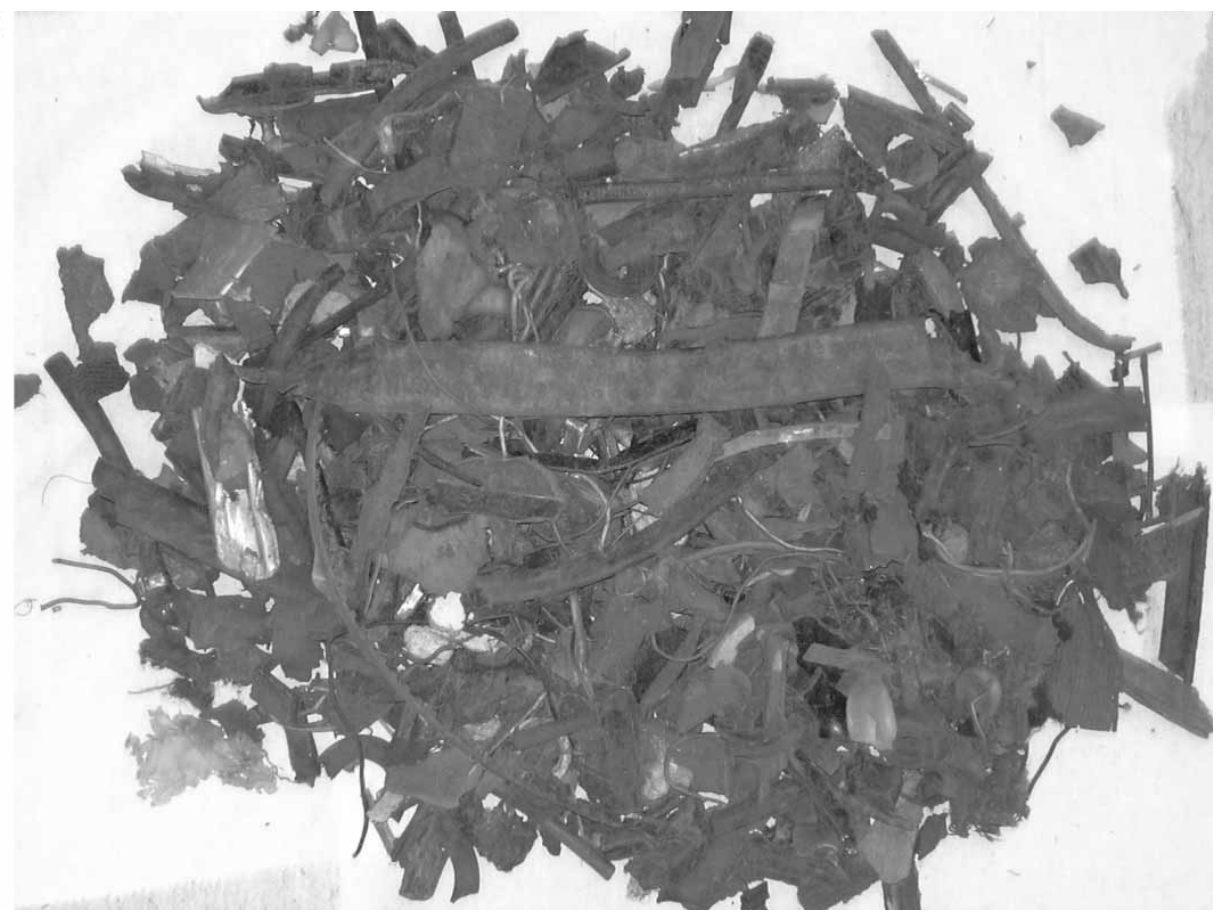

FIGURE 3 Continued.

the main organic components, such as plastics, rubber, wood, foam, etc. The shape effect, which is for effective density separation by other means (e.g. air tables, jigging), is minimal for heavy medium separation. Density separation was investigated using a dry sand fluidized bed. The medium used in the fluidized bed was sand and it was fluidized with air, giving a stable suspension of fine particles with an effective density of around $1400 \mathrm{~kg} / \mathrm{m}^{3}$. The wires were supposed to sink, while the light material, like foam, rubber and some types of plastic, was supposed to float [14]. Wet heavy medium separation was not investigated because it is considered less cost effective compared to dry processing techniques. Waste water must be treated, sludge must be disposed and all the material must be dried after the separation.

\subsubsection{Trommel Screen}

A trommel screen was used to screen the samples and so remove the largest particles. The screening surface is formed into a cylinder mounted on a rotating shaft. The material that must be screened is delivered inside the trommel at one end. The fine material and long objects, in this case the wires, pass through the holes, the coarse material is delivered at the other end.

The mesh used was hexagonal, with two different sizes: 3 and $1.5 \mathrm{~cm}$ side lengths.

\subsubsection{Eddy Current Separation}

The material is fed onto the conveyor belt of an eddy current separator. It is conveyed over a spinning magnetic rotor where separation occurs. The two streams of material 
discharge under and away from the magnetic rotor. The main component of an eddy current separator is the magnetic rotor: it consists of permanent earth magnets mounted in a series to a plate, which in turn is attached to the motor driven shaft. These magnets are covered by a shell, which allows the conveyor belt to ride over it. A stainless steel cover that encapsulates the magnetic rotor is inside the shell, spinning independently from the shell and the belt. When a non-ferrous metal product passes over the shell, the spinning magnets inside the shell generate an eddy current in the metal, thus creating a magnetic field around the piece of metal. The polarity of the magnetic field of the metal is the same as the rotating magnets, causing the metal to be repelled away from the separator. Non-metallic products such as plastics, glass, wires or other process materials simply fall off the end of the separator [15].

Since a very little amount of non-ferrous metals was contained in our samples, this separation only enabled separation of the material into two fractions, according to its weight and shape. A Bakker Magnetics eddy current device was used for the tests. The splitter was positioned near the end of the separator $(1.5 \mathrm{~m})$, the speed of the belt was about $1.7 \mathrm{~m} / \mathrm{s}$ and the rotor speed was $3000 \mathrm{rpm}$.

\subsubsection{Magnetic Separation}

Magnetic separation was carried out using an eddy current separator: the steel is attracted by the magnet and remains on the conveyor belt. The weakly magnetic material feels the magnetic attraction and falls near the magnetic rotor. The nonmagnetic material is driven only by gravity and simply falls off the end of the separator following a parabolic trajectory.

\subsection{Objective of the Technology}

The aim of the tests carried out was to find a cost-effective way to recover wires with resultant high grade and high recovery. The methods mentioned above were investigated to achieve this. Based on the results during the final tests attention was focused on removing the wires using screen and nail roll.

\section{ASR CHARACTERIZATION}

The tests were carried out using three sample of heavy ASR. Each of them is the residue arising from magnetic separation, which was used to recover steel. The sample pictures are shown in Figure 3.

The first sample was used to investigate the efficiency and operational settings of the nail roll. It is not entirely representative of the industry: it was used to find the optimum setting for the nail roll separator.

The second sample tested is ASR from the Italian car scrap recycling industry obtained after heavy medium separation $\left(\sin \mathrm{k}+1850 \mathrm{~kg} / \mathrm{m}^{3}\right)$.

The third sample is also ASR from the Italian car scrap recycling industry. It was divided into two fractions to obtain more results: one smaller $(4 \mathrm{~kg})$, (a) and one larger $(9 \mathrm{~kg})$, (b) fractions.

The wires contained in the first and in the second sample were divided in three classes according to their length: $-5 \mathrm{~cm}, 5-10 \mathrm{~cm},+15 \mathrm{~cm}$. The composition and the properties of these three samples are summarized in Table II. 
TABLE II Composition of the samples

\begin{tabular}{|c|c|c|c|c|c|}
\hline & & \multirow[t]{2}{*}{$1 s t$} & \multirow[t]{2}{*}{$2 n d$} & \multicolumn{2}{|c|}{$3 r d$} \\
\hline & & & & $a$ & $b$ \\
\hline \multicolumn{6}{|l|}{ Composition $(\%)$} \\
\hline Stone and Glass & & 21.8 & 42.5 & 3.4 & 4.4 \\
\hline Plastic and Rubber & & 60.8 & 12.4 & 69.6 & 78.5 \\
\hline Foam/Textiles & & 0 & 0 & 9.1 & 6.6 \\
\hline Wood & & 0 & 0 & 7.6 & 2.5 \\
\hline Metals & & 2.9 & 17.1 & 2.1 & 2.5 \\
\hline \multirow[t]{2}{*}{ Wires } & Tot & 14.5 & 28.0 & & \\
\hline & -5 & 14.1 & 16.9 & & \\
\hline \multirow[t]{2}{*}{ Wires length (cm) } & $5-15$ & 60.0 & 66.1 & 8.4 & 6.1 \\
\hline & +15 & 25.9 & 17.0 & & \\
\hline Average particles size $(\mathrm{cm})$ & & 1 & $3-7$ & $0-5$ & $0-5$ \\
\hline Total weight $(\mathrm{kg})$ & & 4.41 & 8.52 & 4.10 & 9.09 \\
\hline
\end{tabular}

\section{EXPERIMENTAL PROCEDURE}

Results are calculated in terms of 'recovery' and 'grade' indices. Recovery is the percentage of wires that reaches the separated fraction compared to the total amount of wires.

$$
\text { Recovery Index }=\frac{S \cdot 100}{S+L}(\%)
$$

The recovery index of the three length classes in which wires were divided, was calculated dividing the weight of the separated wires belonging to one class by the total weight of the going out wires of that class:

$$
(\text { Recovery Index })_{\text {CLASS }}=\frac{S_{\text {CLASS }} \cdot 100}{S_{\text {CLASS }}+L_{\text {CLASS }}}(\%)
$$

Grade is the percentage of wires in the separated fraction compared to the total wires and non-wires of this fraction.

$$
\text { Grade Index }=\frac{S \cdot 100}{S+S n w}(\%)
$$

The grade index of the three classes in which wires were divided, was calculated dividing the weight of the separated wires belonging to one class by the weight of the separated material as a whole:

$$
(\text { Grade Index })_{\text {CLASS }}=\frac{S_{\text {CLASS }} \cdot 100}{S+S n w}(\%)
$$

The recovery and the grade indices of non-wires were calculated as follows: 


$$
\begin{gathered}
(\text { Recovery Index })_{\text {NON-WIRES }}=\frac{S n w \cdot 100}{S n w+L n w}(\%) \\
(\text { Grade Index })_{\text {NON-WIRES }}=\frac{S n w \cdot 100}{S n w+L n w}(\%)
\end{gathered}
$$

where $S=$ 'wires separated', wires which ended up in the separated fraction; $\mathrm{S}_{\text {CLASS }}=$ separated wires belonging to a class of length; $L=$ 'lost wires', which did not end up in the separated fraction; $L_{\text {CLASS }}=$ lost wires belonging to a class of length; $S n w=$ 'separated non-wires', plastics, rubber and other materials that ended up in the separated fraction with wires; $L n w=$ 'Lost non-wires', plastics, rubber and other materials that are contained in the not separated fraction after separation.

Different settings of the nail roll were tested with the three samples described in Table II in order to achieve the best results. For each setting recovery and the grade indexes were determined.

Figure 4 is a schematic representation of the processes used with the three studied samples. The applied techniques, which are linked by arrows, are listed in chronological order.

\section{RESULTS}

The settings, the results and the techniques to be used to separate the wires depended above all on the property of the material.

\subsection{First Sample}

The wires contained in this sample were divided by hand sorting into three fractions according to their length: $-5 \mathrm{~cm}, 5-15 \mathrm{~cm},+15 \mathrm{~cm}$. Each fraction was painted for an easier distinction after the tests. The sample was directly processed with the nail roll, without any pre-treatment. Different settings were studied in order to find the best results. In every test carried out with this sample the nail distance was $2.75 \mathrm{~cm}$. This value was determined, because it is optimized for the size of the particles.

The achieved recovery index is around $70 \%$ as the average for all wire lengths. It is higher than $90 \%$ for wires longer than $15 \mathrm{~cm}$, while it decreases for the shorter ones. The grade index of the wire product was between 80 and $100 \%$. The best setting is a horizontal distance between the end of the feeder and the centre of the roll of $8 \mathrm{~cm}$ and roll speed of $0.5 \mathrm{rpm}$ (Fig. 5).

For this first sample, due to its limited weight, it was also possible to make 10 tests with the same best setting in order to calculate the average grade and recovery and the standard deviation ( $1.5 \%$ for the grade index and $1.9 \%$ for the recovery index). Figure 5 shows the separation quality in these tests.

\subsection{Second Sample}

The wires of the second sample were also divided into three fractions according to their length. At first, the nail distance was changed because this sample contained a lot of pieces larger than the distance itself and the sample was processed with the nail roll 
First sample

Nail roll
$(1=2.75 \mathrm{~cm})$

Nail roll

$(1=5.5 \mathrm{~cm})$

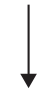

Nail roll

$(1=2.75 \mathrm{~cm})$

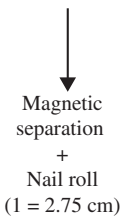

Third sample

First part:

Nail roll

Screen + Nail roll $(1=5.5 \mathrm{~cm})$
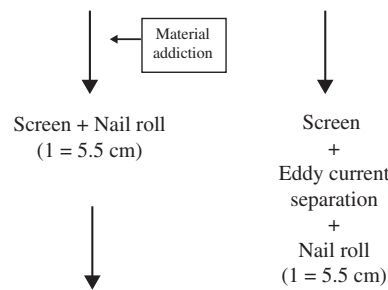

Screen

Density separation

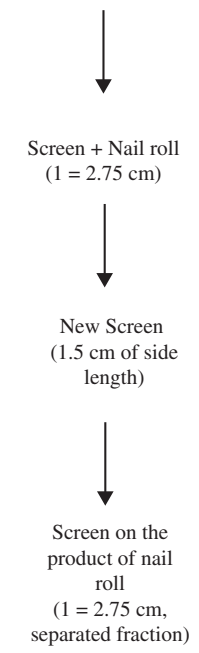

FIGURE 4 Flow chart for the separation methods used on the samples.

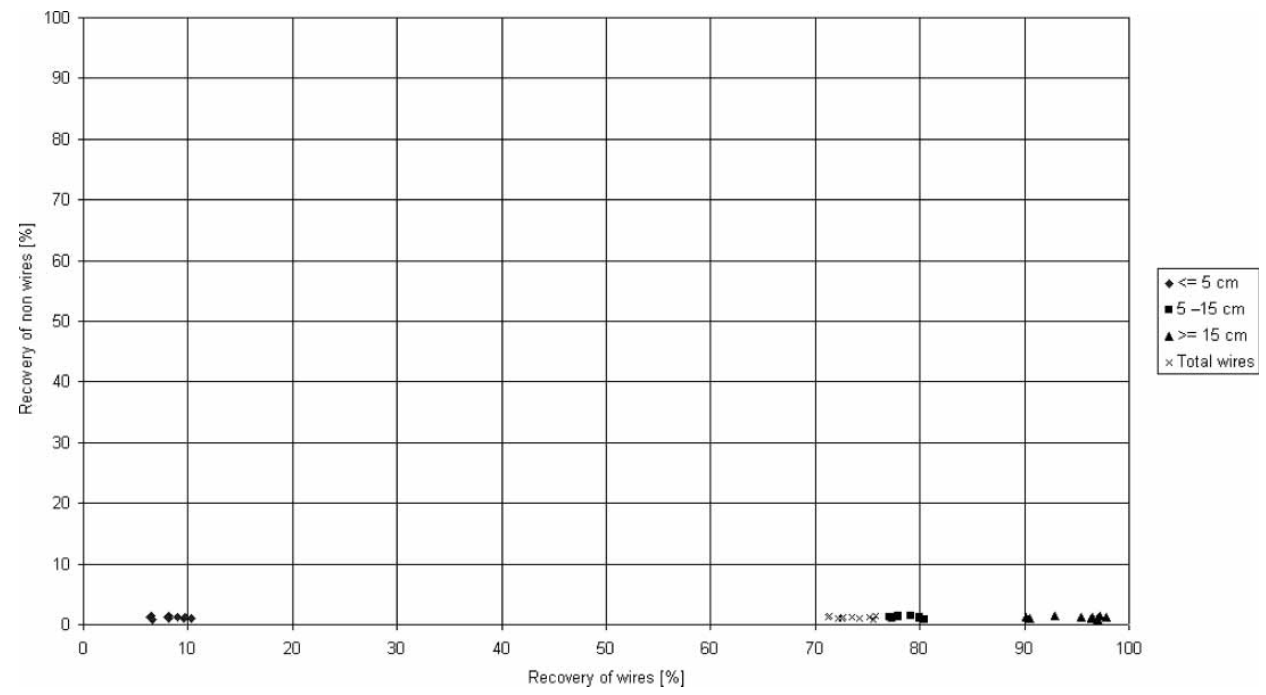

FIGURE 5 Recovery of wires index versus recovery of non-wires index for the best setting. 
directly. Some tests were carried out with a nail distance of $5.5 \mathrm{~cm}$, but the total recovery was lower than for the first sample (less than $60 \%$ in the best case). The grade was between 65 and $75 \%$. The best results were obtained with a distance between the feeder and the center of the roll of $8 \mathrm{~cm}$ and a roll speed of $1.5 \mathrm{rpm}$.

Additional tests were conducted with this setting, while the feeder speed was changed in order to estimate the capacity of the nail roll. The feeder used was $0.30 \mathrm{~m}$ wide and due to its characteristics, four speeds were possible, the fourth speed was the maximum. For each speed the time taken to process the whole sample was recorded and this capacity was calculated as:

$$
\text { Capacity }=\frac{\text { Total weight }[\mathrm{kg}]}{\text { Time clocked }[\mathrm{s}]} \cdot \frac{3600 \mathrm{~s}}{1 \mathrm{~h}}(\mathrm{~kg} / \mathrm{h})
$$

The capacity per meter of feeder width can be determined as follows:

$$
\text { Capacity } / \text { meter }=\frac{\text { Capacity }[\mathrm{kg} / \mathrm{h}]}{\text { Feeder width }[\mathrm{m}]}=\frac{\text { Capacity }[\mathrm{kg} / \mathrm{h}]}{0.3 \mathrm{~m}}(\mathrm{~kg} /(\mathrm{h} \mathrm{m}))
$$

The results are shown in Table III.

To gain better results the nail roll was modified: the nail distance was reduced to $2.75 \mathrm{~cm}$ and a new splitter was used. A new series of tests was carried out in order to find the best setting, which was established as having a distance between the feeder and the center of the roll of $10 \mathrm{~cm}$ and a roll speed of $1 \mathrm{rpm}$.

Magnetic separation was conducted on this sample, because it contained 17\% metals. The sample was then processed with the nail roll. The results obtained after this treatment are as follows: a recovery index that covers a range from 64 to $87 \%$ and a grade index from 70 to $85 \%$. One third of wires were lost during the magnetic separation, because the wires appeared to be weakly magnetic (Table III).

\subsection{Third Sample}

Almost all the wires contained in this sample had lengths between 5 and $15 \mathrm{~cm}$ and the percentage of them in the total composition of the sample was quite low $(6-7 \%)$. For these reasons and to accelerate the tests, they were not divided in classes of length.

The first part of the third sample was initially treated using the nail roll with a high nail distance $(5.5 \mathrm{~cm})$. To improve the grade the removal of the largest pieces of rubber and plastics was tried using the trommel screen with $3 \mathrm{~cm}$ mesh: $2.5 \mathrm{~kg}$ of material of the same composition were added to the sample and the sample obtained in this way was treated with the screen.

The undersize fraction was then processed with the nail roll. The results showed that the grade index actually improved, but it was still below $50 \%$. Density separation was applied after screening using the sand fluidized bed.

A new, heavier fraction of the same sample was taken to verify the efficiency of the trommel screen (second part of the third sample). This fraction was then processed with the nail roll. The following step was to use the eddy current separation after screening this fraction. The light material, mostly foam and light plastics, fell near the conveyor belt, because of the air resistance. The heavy part, made up of light shape plastics, 
TABLE III Recovery and grade indices for the second sample with different feeder speeds depending on the length distribution in the wire fraction $d=8 \mathrm{~cm}$, speed $=1.5 \mathrm{rpm}$

\begin{tabular}{|c|c|c|c|c|c|c|}
\hline Length of wires & $\leq 5 \mathrm{~cm}$ & $5-15 \mathrm{~cm}$ & $\geq 15 \mathrm{~cm}$ & Total wires & Non-wires & Total \\
\hline \multicolumn{7}{|l|}{ Feeder speed $1 \Rightarrow$ TIME: $19^{\prime} 39^{\prime \prime}$} \\
\hline Fraction not separated (kg) & 0.28 & 0.64 & 0.06 & 0.97 & 5.33 & \\
\hline Fraction separated $(\mathrm{kg})$ & 0.03 & 0.88 & 0.34 & 1.25 & 0.47 & \\
\hline Total $(\mathrm{kg})$ & 0.31 & 1.51 & 0.40 & 2.22 & 5.80 & 8.02 \\
\hline Recovery $(\%)$ & 10.25 & 57.90 & 85.29 & 56.17 & & \\
\hline Grade $(\%)$ & 1.85 & 51.04 & 19.82 & 72.72 & & \\
\hline Capacity $(\mathrm{kg} / \mathrm{h})$ & & & & & & 24.49 \\
\hline Capacity/meter (kg/(h m)) & & & & & & 81.63 \\
\hline \multicolumn{7}{|l|}{ Feeder speed $2 \Rightarrow$ TIME $: 6^{\prime} 49^{\prime \prime}$} \\
\hline Fraction not separated (kg) & 0.26 & 0.60 & 0.02 & 0.88 & 5.33 & \\
\hline Fraction separated $(\mathrm{kg})$ & 0.04 & 0.94 & 0.33 & 1.31 & 0.58 & \\
\hline Total $(\mathrm{kg})$ & 0.31 & 1.54 & 0.35 & 2.19 & 5.91 & 8.10 \\
\hline Recovery $(\%)$ & 13.68 & 61.18 & 93.93 & 59.78 & & \\
\hline Grade $(\%)$ & 2.22 & 49.80 & 17.47 & 69.49 & & \\
\hline Capacity $(\mathrm{kg} / \mathrm{h})$ & & & & & & 71.32 \\
\hline Capacity/meter $(\mathrm{kg} /(\mathrm{h} \mathrm{m}))$ & & & & & & 237.74 \\
\hline \multicolumn{7}{|l|}{ Feeder speed $3 \Rightarrow$ TIME: $2^{\prime} 59^{\prime \prime}$} \\
\hline Fraction not separated $(\mathrm{kg})$ & 0.23 & 0.40 & 0.01 & 0.64 & 4.81 & \\
\hline Fraction separated $(\mathrm{kg})$ & 0.08 & 1.11 & 0.37 & 1.55 & 0.78 & \\
\hline Total $(\mathrm{kg})$ & 0.31 & 1.51 & 0.38 & 2.19 & 5.59 & 7.78 \\
\hline Recovery $(\%)$ & 24.92 & 73.43 & 97.61 & 70.79 & & \\
\hline Grade $(\%)$ & 3.27 & 47.51 & 15.72 & 66.50 & & \\
\hline Capacity $(\mathrm{kg} / \mathrm{h})$ & & & & & & 156.51 \\
\hline Capacity/meter $(\mathrm{kg} /(\mathrm{h} \mathrm{m}))$ & & & & & & 521.68 \\
\hline \multicolumn{7}{|l|}{ Feeder speed $4 \Rightarrow$ TIME: $1^{\prime} 50^{\prime \prime}$} \\
\hline Fraction not separated $(\mathrm{kg})$ & 0.11 & 0.26 & 0.00 & 0.37 & 2.86 & \\
\hline Fraction separated $(\mathrm{kg})$ & 0.19 & 1.26 & 0.36 & 1.81 & 2.68 & \\
\hline Total $(\mathrm{kg})$ & 0.30 & 1.52 & 0.36 & 2.18 & 5.53 & 7.72 \\
\hline Recovery $(\%)$ & 63.09 & 82.98 & 99.61 & 83.00 & & \\
\hline Grade $(\%)$ & 4.23 & 28.08 & 8.04 & 40.34 & & \\
\hline Capacity $(\mathrm{kg} / \mathrm{h})$ & & & & & & 252.51 \\
\hline Capacity/meter $(\mathrm{kg} /(\mathrm{h} \mathrm{m}))$ & & & & & & 841.70 \\
\hline
\end{tabular}

wires, stones actually fell after the splitter, following a parabolic trajectory. This part was then processed with the nail roll. Eddy current separation enabled to remove around $32 \%$ of non-wires, with a recovery of about $80 \%$. The series of tests executed with the same sample before and after eddy current separation show about the same results.

To improve the recovery, the nail distance was reduced to $2.75 \mathrm{~cm}$ and a new splitter was employed. The results of the series of tests carried out with this new setting show a recovery index in a range from 65 to $85 \%$, but a grade below $40 \%$.

To improve the grade two more solutions were proposed: to process the feed of the nail roll in a trommel screen with a smaller mesh $(1.5 \mathrm{~cm}$ of side length) and to process the best product of the nail roll in the trommel screen with a larger mesh $(3 \mathrm{~cm})$. With the first solution the wire recovery index after screening was only $52 \%$. The second solution improved considerably the grade index, which rose from 30 to $85 \%$, but deteriorated the recovery index, which decreased from 88 to $53 \%$.

All the results obtained with the samples are summarized in Table IV. The number in brackets indicating the pre-treatment refers to Fig. 6. 
TABLE IV Results

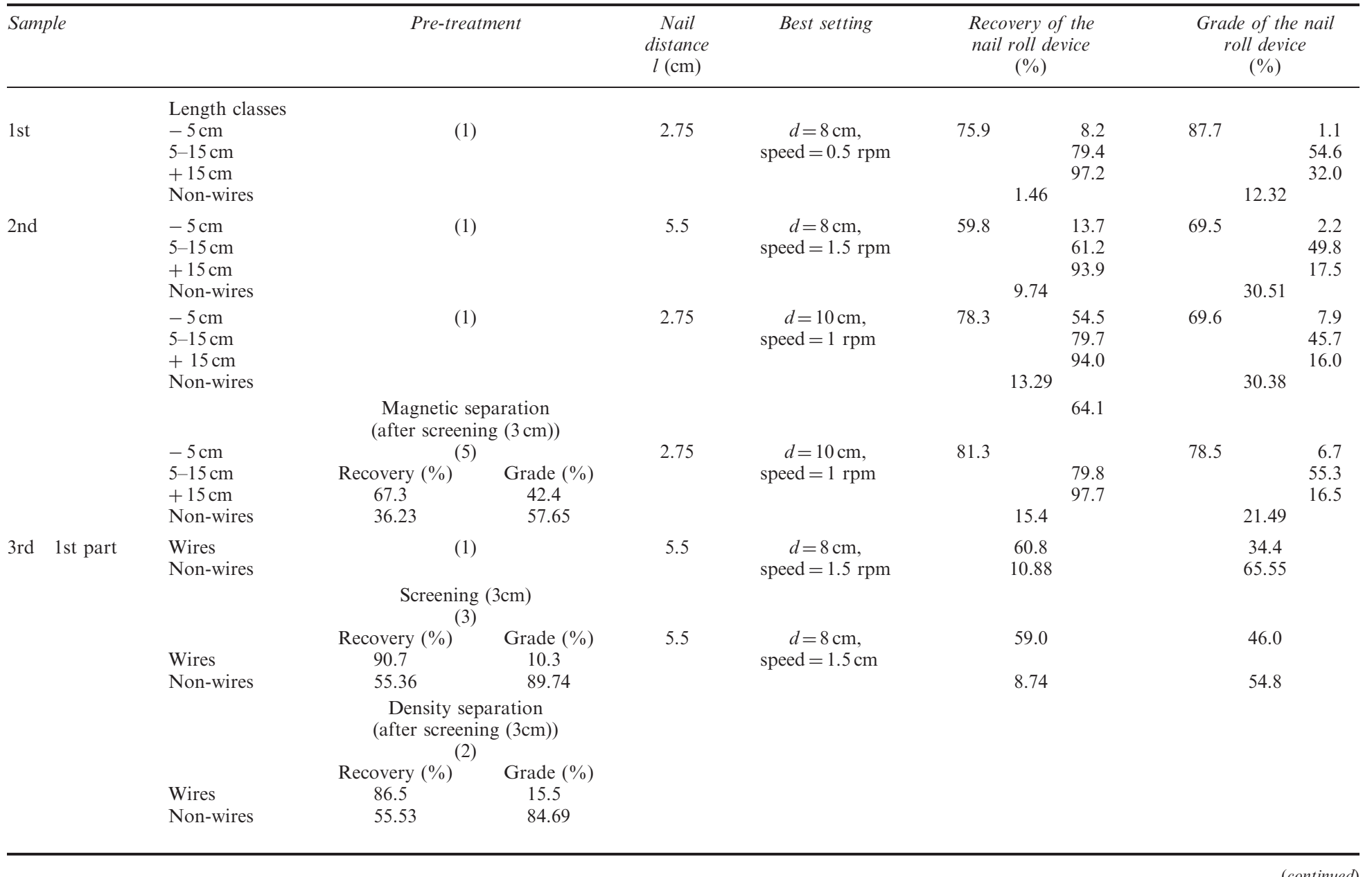


TABLE IV Continued

\begin{tabular}{|c|c|c|c|c|c|c|c|}
\hline Sample & & \multicolumn{2}{|c|}{ Pre-treatment } & \multirow{2}{*}{$\begin{array}{c}\text { Nail } \\
\text { distance } \\
l(\mathrm{~cm})\end{array}$} & \multirow[t]{2}{*}{ Best setting } & \multirow{2}{*}{$\begin{array}{c}\text { Recovery of the } \\
\text { nail roll device } \\
(\%)\end{array}$} & \multirow{2}{*}{$\begin{array}{c}\text { Grade of the nail } \\
\text { roll device } \\
(\%)\end{array}$} \\
\hline \multirow[t]{10}{*}{ 2nd part } & & \multicolumn{2}{|c|}{$\begin{array}{l}\text { Screening }(3 \mathrm{~cm}) \\
(3)\end{array}$} & & & & \\
\hline & & Recovery (\%) & Grade $(\%)$ & \multirow[t]{3}{*}{5.5} & \multirow{3}{*}{$\begin{array}{c}d=10 \mathrm{~cm} \\
\text { speed }=2 \mathrm{rpm}\end{array}$} & 55.4 & 43.0 \\
\hline & Wires & 90.2 & 12.1 & & & & \\
\hline & Non-wires & 47.23 & 87.96 & & & 9.49 & 57.03 \\
\hline & & \multicolumn{2}{|c|}{$\begin{array}{c}\text { ECS (after screening }(3 \mathrm{~cm})) \\
(4)\end{array}$} & & & & \\
\hline & Wires & $\begin{array}{l}\text { Recovery }(\%) \\
82.7\end{array}$ & $\begin{array}{l}\text { Grade }(\%) \\
\quad 13.0\end{array}$ & \multirow[t]{2}{*}{5.5} & \multirow[t]{2}{*}{$\begin{array}{c}d=10 \mathrm{~cm} \\
\text { speed }=2 \mathrm{rpm}\end{array}$} & 56.2 & 50.2 \\
\hline & Non-wires & 62.12 & 86.87 & & & 8.48 & 49.79 \\
\hline & & \multicolumn{2}{|c|}{$\begin{array}{l}\text { Screening }(3 \mathrm{~cm}) \\
(3)\end{array}$} & & & & \\
\hline & Wires & $\begin{array}{l}\text { Recovery }(\%) \\
90.2\end{array}$ & $\begin{array}{l}\text { Grade }(\%) \\
\quad 12.1\end{array}$ & 2.75 & $\begin{array}{c}d=10 \mathrm{~cm}, \\
\text { speed }=1.5 \mathrm{rpm}\end{array}$ & 76.7 & 35.5 \\
\hline & Non-wires & 47.23 & 87.96 & & & 19.35 & 64.46 \\
\hline \multirow[t]{8}{*}{ 3rd part } & & \multicolumn{2}{|c|}{ Screening $(1.5 \mathrm{~cm})$} & & & & \\
\hline & & Recovery (\%) & Grade $(\%)$ & & & & \\
\hline & Wires & 47.1 & 30.0 & & & & \\
\hline & Non-wires & 13.87 & 69.89 & & & & \\
\hline & & \multicolumn{2}{|c|}{$\begin{array}{l}\text { Screening }(3 \mathrm{~cm}) \\
\text { of the nail roll product } \\
\text { (3) }\end{array}$} & & & & \\
\hline & & Recovery $(\%)$ & Grade $(\%)$ & & & & \\
\hline & & 53.4 & 85.7 & & & & \\
\hline & & 5.88 & 18.32 & & & & \\
\hline
\end{tabular}

Notes: (1) No pre-treatment; (2) Pre-treatment: screening + density separation; (3) Pre-treatment: screening; (4) Pre-treatment: screening + eddy current separation; (5) Pre-treatment: screening + magnetic separation. 


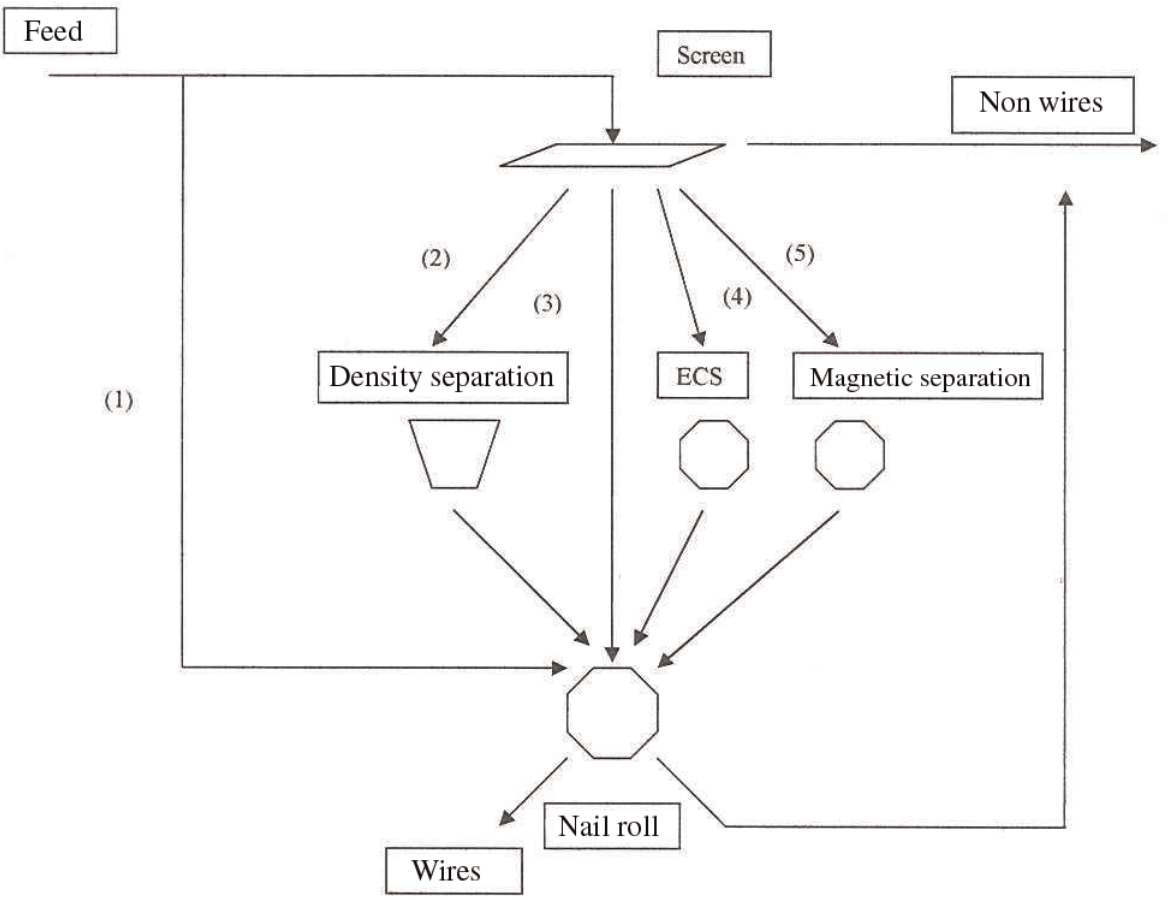

FIGURE 6 Schematic representation of the system trommel screen + sand fluidized bed or ECS or magnetic separation + nail roll.

\section{DISCUSSION}

\subsection{First Sample}

The first results, achieved using the nail roll on the ASR directly, were fairly good, so it was not necessary to apply other separation techniques. The recovery was higher for the longest wires because they are more easily captured by the nails.

\subsection{Second Sample}

It can be observed that as the feeder speed increases, the removal capacity and the recovery index increase, while the grade decreases. This occurs because when using a high feeder speed many wires as well as non-wires end up in the separated fraction, causing a decrease of the grade index and an increase of the recovery index.

The recovery index was better with a nail distance of $2.75 \mathrm{~cm}$ than with a distance of $5.5 \mathrm{~cm}$ : it is higher than $60 \%$ not only for the longest wires, but for every wire longer than $5 \mathrm{~cm}$. The grade was just a few percentage points lower than that achieved in the previous series of tests, because of the presence of the second splitter.

Magnetic separation proved to be useful only for the removal of steel content: both the weakly magnetic and the non-magnetic fractions contained a high percentage of wires: $33 \%$ of wires proved to be weakly magnetic. It can be concluded that it was not effective. 


\subsection{Third Sample}

Applying the trommel screen with the first mesh ( $3 \mathrm{~cm}$ of side length) almost all the wires fell to the undersize, obtaining a high recovery index $(90 \%)$, approximately $50 \%$ in weight of non-wires was removed. The second mesh $(1.5 \mathrm{~cm}$ of side length) was not useful because around $50 \%$ of wires ended up in the oversize.

After density separation using a dry sand fluidized bed, wire recovery proved to be low: $86 \%$ removing only $44 \%$ of the non-wires. This result is good if obtained using a trommel screen, but not using density separation: it requires a more complex technology and it is more expensive. Moreover the concentration of the wires remained almost the same before and after this separation (about 13\%).

Eddy current separation displayed the same problems as density separation: low wire recovery (about 80\%), low non-wire removal (only 31\%), and therefore not effective.

As for the second sample, the recovery index was better with a nail distance of $2.75 \mathrm{~cm}$ than with $5.5 \mathrm{~cm}$ : it is $34 \%$ higher in the best case. However the grade index is lower: lower by $46 \%$ in the worst case.

For the material of this sample some further solutions need to be found to improve the grade after trommel screening and nail roll separation.

\subsection{Economic Aspects}

The objective of mechanical concentration is to increase profit of the entire processing chain. In the example of car processing it means achieving an optimized recovery of raw materials and energy at the lowest possible cost. Therefore an optimized mechanical ASR pre-treatment process offers a balance between grade, recovery and cost, instead of being solely an attempt to obtain the purest products at the highest recovery. Finding this balance falls outside the scope of this work; however, some important factors are mentioned here that may assist in a proper economic positioning of the investigated technology.

\subsubsection{Advantages}

A nail roll/screening process is entirely dry; therefore there are no sludge treatment, wet circuit and additives or medium costs. Nothing is added to the processed material. Additioanlly, the absence of active airflow prevents dust generation.

The mechanical construction of the nail roll separator is simple. A major part of the system can be constructed by combining existing equipment: belt roll drive, vibratory conveyor, feeder and splitter planes. Only the roll itself and its frame, which is mounted on the vibratory conveyor, must be newly designed.

Of all investigated dry technologies so far the nail roll has the best performance for the pre-concentration of electric wires from mixtures.

Assuming an average grade of $70 \%$, of each tonne of wire product it is estimated that between 100 and $200 \mathrm{~kg}$ of copper/aluminium mixture and approximately $400-600 \mathrm{~kg}$ of PVC and flame retardant plastics are removed. This may significantly increase thermal utilization opportunities of the remaining material, while the metal value of the separated wire fraction compensates the treatment/landfill costs of the $\mathrm{PVC} /$ flame retardant residue component of the wire fraction. 


\subsubsection{Disadvantages}

Referring to Table III capacity of not more than $1 \mathrm{t} / \mathrm{h}$ per meter roll width can be achieved. Roll widths of over $2 \mathrm{~m}$ may be unfavorable from a mechanical perspective, hence requiring multiple separators for feeds in excess of $2 \mathrm{t} / \mathrm{h}$.

The grade and recovery of wires of poorer mixtures (e.g. around $10 \%$, sample 3 ) is rather low. The system is originally designed for non-fluffy NF containing mixtures. Excess presence of fluffy material in ASR (foams, textiles) reduces the effectiveness of the system.

Only wires that are longer than the average particle size can be separated.

There may be occasional sticking of objects between the nails, which may require specific attention, and regular cleaning.

Taking the above into consideration and referring to the experimental results presented in this work it is assumed that a combination of a staged trommel screen and nail rolls that are optimized for each size fraction is the most effective industrial configuration. In this way the disadvantage of the capacity of the nail rolls is removed by treating well classified size ranges that due to trommel screening are already pre-concentrated with longer wires. The wire fractions of each roll can be successively combined. In case excessive sticking may occur, a simple pneumatic valve blowing pressurized air along the nails at regular time intervals may be a cost effective solution.

\section{CONCLUSION}

The problem of ELV disposal has become very real in the last few years and more and more efforts are made all worldwide in order to reduce its impact on the environment, recovering materials and energy. This is also a result of the issue of the EU Directive 2000/53/EC that states that every car manufacturer shall collect, recover and recycle its ELV before 2006.

The project of the nail roll separator aims to find a cost-effective method for the recovery of a valuable fraction of the heavy residue after shredding: aluminium and copper wires.

Different methods, including various pre-treatments, were tested and it can be concluded that magnetic, density and eddy current separation are not cost effective: minor improvements do not justify the higher losses and additional processing efforts. In the realized tests $33 \%$ of wires are lost by magnetic separation, $14 \%$ by density separation with sand fluidized bed and $18 \%$ by eddy current separation. On the other hand, screening by trommel screen proved to be efficient: it is cost effective and it gives wire recovery of $90 \%$.

Nail roll efficiency is mostly dependent on the properties of the material treated and on the roll setting based on these properties. The recovery index is higher than $70 \%$ in the best case for all three tested samples, while the grade index in the best case is $87 \%$ for the first sample, $70 \%$ for the second and $35 \%$ for the third.

It can be concluded from this overview of methods for separating wires from ASR that for future investigation the attention has to be focused on further development of trommel screening and nail roll separation. 


\section{References}

[1] N. Fraunholcz, E.A. Schokker, P.C. Rem and W.L. Dalmijn, Mechanical pre concentration of ASR, International Automobile Recycling Congress, Geneva, Switzerland, 13-15 March, 2002.

[2] J. Dillmann and A. van der Beek, Explotation and preparation possibilities of ASR, Proceedings of the XX IMPC-Aachen, 5 (1997), 201-208.

[3] C.A. Ambrose, M.M. Singh and M.K. Harder, The Material Composition of Shredder Waste in the UK, Waste and Energy Research Group, School of the Environment, University of Brighton, Brighton, 2000.

[4] Proposal for a council directive on end of life vehicles, The End of Life Directive, 25 June 1999, pp. 1-10.

[5] Chlorine chemistry council - Chlorine Disinfects: PVC in Vehicles, URL: (www.c3.org:80/library/ pveveh.html $\rangle$.

[6] Copper waste recycling; Copper prices; supply and demand, 'Copper at a Crossroads', Recycling Today, 40(4) (2002), 26, 28-30.

[7] Automotive Application: What's Ahead in 2007?, February 98, URL: 〈http://innovations.copper.org .

[8] A.A. Nijkerk and W.L. Dalmijn, Handbook of Recycling Techniques, Nijkerk Consultancy, The Hague, 2001.

[9] B.J.N. Linger and C. Verschut, 'Recycling on ferrometaal/kunststofcombinaties (NFCK's)', Eindrapport deel 3: Werkgebied van droge deeltjes scheidingsapparauur. TNO-report R95-032, TNO-MEP, Apeldoorn, 1995.

[10] K. Löhr, M. Melchiorre and B. Kettemann, U. Aufbereitungstechnik, Carl Hanser Verlag, München, 1995.

[11] V. Herman, Anwendung des Dryflo-Wirbelbettverfahrens zur Zerlegung von Kupferkabel-Schrott', Erzmetall Bd.24, 12 (1971), 584-587.

[12] T.P.R de Jong, 'Separating device', International patent WO 01/64357 A1.

[13] E.M. Beunder and J.P. Rendorp 'Wire separation report', Delft University of Technology, Delft, 30 June 2000 .

[14] T.P.R de Jong, R.A. Felix and W.L. Dalmijn, Dry density separation of 4-16 mm non ferrous car scrap, Proceedings of the XX IMPC-Aachen, 5 (1997), 189-200.

[15] P.C. Rem, Eddy Current Separation, Eburon, Delft, 1999.

\section{BIOGRAPHIES}

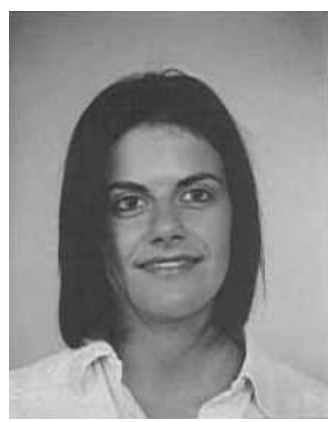

Lara Fabrizi was born in 1977. She graduated with full marks in environmental engineering at the University of Trieste in July 2003. Since October 2002 she spent six months at the Department of Applied Science of the Delft University of Technology.

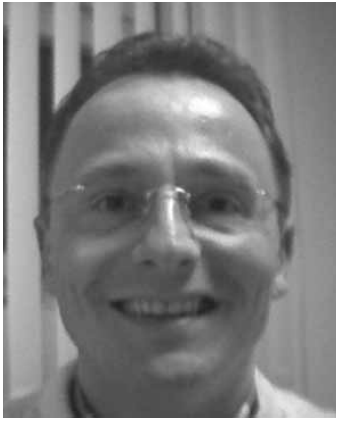

Paolo Bevilacqua was born in Trieste in 1962. He is a professor of raw materials engineering at the University of Trieste. His main area of interests are: ore dressing and recycling process technologies. He is an author of approximately 80 papers. He belongs to the International Committee of Scientific Congresses. 


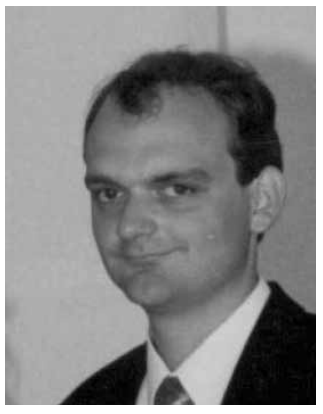

Tako P.R. de Jong was born in 1970 . He is a lecturer in mineral processing at Delft University of Technology. His research topics are: solid fuel preparation, recyclable materials, sensor sortation, automatic quality control, physical concentration methods for solid particles. Mr. de Jong is an author of several journal papers. 